P4 A CRITICAL COMPARISON OF THREE DIAGNOSTIC TECHNIQUES USED FOR THE DETECTION OF TRICHOMONAS VAGINALIS (TV)

Emma Parker*, Henna Jaleel, Mohd Sabri Abu Bakar. Southend University Hospital NHS Trust, Essex, UK

\subsection{6/sextrans-2015-052126.48}

Background British Association of Sexual Health and HIV (BASHH) has recommended that nucleic acid amplification tests (NAAT) to become a gold standard method of TV detection in the 2014 guidelines.

Aims To compare the efficacy of traditional wet mount microscopy (WMM), culture and TMA (Transcription Mediated Amplification) by Aptima assay for the detection of TV in our local population. The cost effectiveness of TMA and staffing requirements will also be assessed.

Methods All female patients with vaginal discharge, male contact patients and males with persistent urethritis were included. Aptima high vaginal/cervical swabs routinely tested for chlamydia or gonorrhoea by TMA were used for Aptima TV testing, as were urines. All swabs and urine samples had WMM performed and cultured in modified diamonds media. Positivity rate, sensitivity, specificity, positive predictive value (PPV) and costs per test were calculated. The statistical significance was measured by McNemars test.

Results 436 patients were included in the study, 64 male and 372 female. 11 were positive by at least one method, including one male. All TMA positive patients were also positive by urine except for 2. There is no statistical difference between WMM and culture $(\mathrm{p}=0.25)$ but a highly significant difference between TMA and culture $(p=0.0124)$ and TMA and WMM $(p=0.0043)$. TMA is the most expensive test at $>£ 5$ per test.

Conclusions TMA is the most sensitive test for TV. It has fast turnaround time and suitable for female urine samples. Its use is limited due to cost and suitability for other samples e.g. in male patients.

\section{P5 GONORRHOEA AUDIT: CHANGING PATTERN OF ANTIBIOTIC SENSITIVITY AND PERSISTENCE OF DNA DETECTION 2007-2014}

Sris Allan*, Jessica Jefferson. Coventry and Warwickshire Partnership Trust, Coventry, UK

10.1136/sextrans-2015-052126.49

Background Nucleic acid amplification testing (NAAT) is widely used in GUM clinics to diagnose GC infection; its in-built high sensitivity may potentially detect DNA from non-viable organisms following successful treatment. The BASHH national guidelines stipulate that test of cure (TOC) with NAAT should take place 2 weeks post treatment. The purpose of this study was to determine whether this is an adequate time interval to perform TOC. We also analysed the changing pattern of antibiotic sensitivity between 2007-2014.

Methods All GC cases at our clinic between $01 / 01$ to $30 / 06$ in 2007-2014 were identified and assessed for antibiotic sensitivity and TOC.

Results In 2014 there were 126 cases, culture and sensitivity results were available for 85 . TOC with NAAT was done in
71 cases. There were 5 cases where the NAAT was SDA positive but not PCR, two of these had a negative NAAT when tested at 29 and 57 days post treatment. Two patients DNA for a repeat NAAT. The fifth had serial repeated NAAT SDA positive results (however original cultures were sensitive to $1^{\text {st }}$ line therapy), this patient had a negative NAAT after re-treatment. There was one positive result 14 days after treatment (re-treated); the NAAT was not repeated. Overall a TOC with NAAT was performed between 7-50 days after treatment with a mean, median and mode of 18,15 and 14 days respectively.

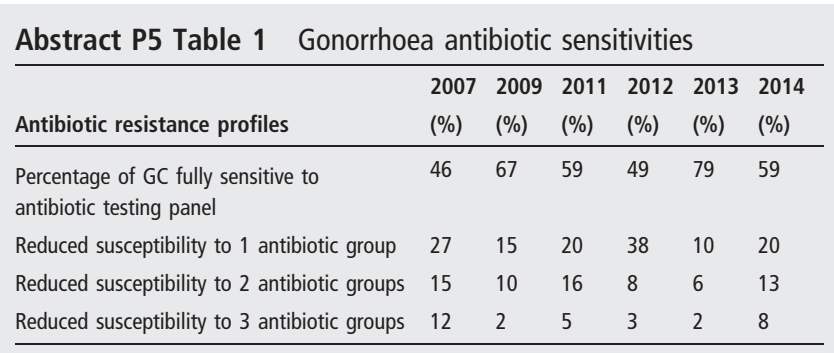

Conclusion None of the cultures were resistant to ceftriaxone. Follow up with NAAT testing at 2 weeks appeared to be adequate for TOC.

\section{P6 LOVE LETTERS - THE NOTIFICATION GAME}

Alex Shaw, Paul Thorne, Arnold Fernandes, Kate Horn*. Royal United Hospital NHS Foundation Trust, Bath, UK

\subsection{6/sextrans-2015-052126.50}

Background In accordance with the national trend there was a perceived increased incidence of anogenital and pharyngeal gonorrhoea presenting to the sexual health $(\mathrm{SH})$ department at a district general hospital (DGH) in the year March 2013-2014.

Aims and objectives To establish the incidence of gonorrhoea in our SH patient population (March 2013-2014). To ensure that the $\mathrm{SH}$ department was compliant with BASHH standards for the management of gonorrhoea.

Methods Retrospective audit of all confirmed cases of gonorrhoea presenting to $\mathrm{SH}$ at one DGH in the period March 2013-2014.

Results 79 patients were identified; all were audited. There was a high level of adherence to BASHH standards but concern regarding partner notification. Partner notification was initiated in $86 \%$ of cases, $95 \%$ were patient led. Patient led referral resulted in poor evidence of partner notification. $71 \%$ of all cases reported more than 1 sexual partner in the previous 3 months yet $57 \%$ of the total cases notified just 1 contact. 4 patients reported 10 or more contacts; only 1 patient was recorded as having notified 10 or more partners.

Discussion/conclusion Poor partner notification may be contributing to increasing gonorrhoea prevalence. It has been suggested that patients from different demographics may preferentially use different, specific communication methods for partner notification. By working with patients to individually tailor notification methods based on demographic background, notification rates may be improved. This is an approach under consideration by $\mathrm{SH}$ at this $\mathrm{DGH}$. 